\title{
A Case Report on Nutritionally Deficient Bacteria: Abiotrophia adiacens
}

\author{
Bethel Shiferaw $^{\mathrm{a}, \mathrm{c}}$, Danielle Morrone ${ }^{\mathrm{a}}$, Ebisa Bekele ${ }^{\mathrm{a}}$, Tabassum Yasmin ${ }^{\mathrm{a}, \mathrm{b}}$
}

\begin{abstract}
Abiotrophia is a nutrition-deficient gram-positive cocci comprising of mainly two species (A. adiacens and $A$. defective). The name Abiotrophia is derived from the Greek language meaning "life nutrition deficiency". Abiotrophia species is part of normal flora of the human upper respiratory, gastrointestinal, and urogenital tracts. It has been associated with serious infections like bacteremia and endocarditis. This paper discusses a case of $A$. adiacens causing bacteremia and vertebral osteomyelitis. A 62-year-old male presented with back pain, fever and chills. Spinal imaging study showed spinal abscess and spinal osteomyelitis, and the blood culture grew A. adiacens. Abiotrophia species, formerly referred to as nutritionally variant streptococci, needs supplemented media for growth and survival. There are extremely few cases that have been reported with spinal involvement due to Abiotrophia species. Resistance to multiple antibiotics has been described in this species. Abiotrophia-associated infections are rare but could be life-threatening. Clinicians should be cognizant of its potential in causing severe infections. We report this case in an attempt to increase awareness about this organism.
\end{abstract}

Keywords: Abiotrophia adiacens; Abiotrophia defective; Life nutrition deficiency; Nutritionally variant; Deficiency; Streptococci; Bacteremia; Vertebral osteomyelitis

\section{Introduction}

Nutritionally variant streptococcus (NVS) was originally described by Frenkel and Hirsch in 1961 [1]. In 1995 a new ge-

\footnotetext{
Manuscript accepted for publication April 29, 2015

aNassau University Medical Center, Department of Internal Medicine, an Affiliation of North Shore Long Island Jewish Health Care System, East Meadow, NY, USA

bNassau University Medical Center, Division of Infection Disease, East Meadow, NY, USA

${ }^{\mathrm{c} C}$ Corresponding Author: Bethel Shiferaw, Nassau University Medical Center, 2201 Hempstead Turnpike, East Meadow, NY 11554, USA.

Email: bshifera@numc.edu
}

doi: http://dx.doi.org/10.14740/jmc2163w nus, Abiotrophia, belonging to NVS was discovered. "Abiotrophia" means life nutrition deficiency because it requires supplemented media for growth and survival [2].

Abiotrophia species form part of the normal flora of the oral cavity, the genitourinary tract, and the intestinal tract [3]. Bacteraemia and endocarditis (5\% of cases) are the more frequently reported clinical infections due to Abiotrophia. Isolated cases of keratitis, endophthalmitis, central nervous system infections, sinusitis, otitis media, prostatitis, cholangitis, arthritis and osteomyelitis have also been reported [4].

Spinal involvement with osteomyelitis due to Abiotrophia is extremely rare. We report a case of bacteremia and vertebral osteomyelitis associated with Abiotrophia adiacens.

\section{Case Report}

A 62-year-old male patient presented with a history of few weeks of constant mid back pain after an accidental fall. He had noticed several episodes of fever and chills associated with the pain. He had no bowel, urinary complaint or other symptoms. He was on medications for his hypertension. The patient did not have any predisposing factors including diabetes mellitus, malnutrition, immune suppression or malignancy. The patient denied any dental procedure or any other surgery done recently. He does not smoke, drink alcohol or use illicit drugs. The patient is retired and lives with his wife. On examination, the lower thoracic spine was tender to firm palpation. The patient did not have any dental caries. Sensory and motor examination was intact in all extremities. The remainder of the clinical examination was grossly unremarkable with no possible source of infection.

The culture from blood was taken, which later grew $A$. adiacens (two out of two bottles). Susceptibility testing later revealed that the bacterium was sensitive to penicillin, oxacillin, ceftriaxone, vancomycin, clindamycin, linezolid and levofloxacin but resistant to gentamicin. Transesophageal echocardiogram (TEE) however ruled out vegetation on the heart valves. The magnetic resonance imaging (MRI) of the spine revealed phlegmonous changes at T10-T12 in the epidural space and paravertebral regions consistent with osteomyelitis and compression fracture at $\mathrm{T} 11$.

A diagnosis of diskitis/osteomyelitis was made based on the imaging results. Laminectomy with fusion surgery of T9- 
L2 vertebrae was done followed by complex wound closure. Bone culture from the T11 vertebral body was sent which revealed no organism. Patient was initially started on ampicillin and later changed to vancomycin as patient developed interstitial nephritis. The patient was treated with 6 weeks of intravenous vancomycin. Patient was transferred to acute rehabilitation unit for restoring baseline functional capacity. His symptoms resolved during his stay in the hospital. The repeat blood culture indicated no bacterial growth. The MRI result after completion of treatment showed fragmented T11 vertebral body and hyperintensity with in the T11 vertebral body.

\section{Discussion}

Abiotrophia species have formerly been referred to as NVS because of their particular nutritional growth requirements [3]. This species should be suspected when Gram stains of specimens reveal microbial cells but cultures are negative. Supplementation of laboratory media is desirable and often necessary to encourage the growth of these organisms [3].

Bacterial pathogens can reach the spine by different routes and hematogenous spread is the most common route. The organisms reach the vertebral body first and then spread to the disk space [5]. At times the presentation could be misleading, as in our patient who came after the fall accident, and one would think fracture as the main differential diagnosis. Therefore obtaining a microbiological diagnosis is mandatory in vertebral osteomyelitis [6].

Spinal infections are commonly caused by Staphylococcus aureus and Streptococcus species, and in approximately one-third of patients the infective organisms cannot be identified [5]. Cases of Abiotrophia infection may be misdiagnosed as culture-negative infections due to its fastidious nature [7]. In our case, two out of two bottles from peripheral blood grew the bacteria, thus laboratory contamination was unlikely.

Literature review indicated that there are extremely few cases that have been reported to date with spinal infection due to Abiotrophia species [6, 8-10]. The organism was identified only from blood culture for almost all cases. In one case, diagnosis was confirmed by identification of $A$. adiacens from infected disk [10]. In our patient, Abiotrophia vertebral osteomyelitis was documented by the growth and culture of the organism from blood.

Different data suggest that NVS account for 5-6\% of microbial endocarditis cases and may be an essential cause of culture-negative endocarditis [3]. Abiotrophia endocarditis carries higher morbidity and mortality than endocarditis caused by other streptococci [3]. Although vegetations seemed to be comparatively small, embolization was common. These infections proved difficult to treat with a relapse rate of $17 \%$ and a bacteriologic failure rate of $41 \%$ [3]. Abiotrophia bacteremia has been reported along with endocarditis in most cases, but in our patient TEE has revealed normal study with no vegetation.

Antimicrobial susceptibility testing of bacterial isolates should be done for detecting drug resistance and selecting appropriate therapy. In those patients with suboptimal response to initial therapy with beta-lactam antibiotics, treatment with vancomycin should be considered [4]. Our patient was treated with 6 weeks of vancomycin. The post-treatment MRI of spine showed signs of osteomyelitis; however, the patient's symptoms improved with antibiotic and rehab services.

\section{Conclusion}

Although infection with Abiotrophia species is rare, clinicians should be aware of this highly aggressive organism. We understand that diagnosing infection with this fastidious microorganism might be challenging, therefore high index of suspicion is essential for adequate diagnosis and treatment. We believe reporting this case would contribute to increasing awareness about this organism among health care professionals.

\section{Conflict of Interests}

The authors declare that there is no conflict of interests regarding the publication of this article.

\section{References}

1. Frenkel A, Hirsch W. Spontaneous development of L forms of streptococci requiring secretions of other bacteria or sulphydryl compounds for normal growth. Nature. 1961;191:728-730.

2. Christensen JJ, Facklam RR. Granulicatella and Abiotrophia species from human clinical specimens. J Clin Microbiol. 2001;39(10):3520-3523.

3. Ruoff KL. Nutritionally variant streptococci. Clin Microbiol Rev. 1991;4(2):184-190.

4. Senn L, Entenza JM, Greub G, Jaton K, Wenger A, Bille $\mathrm{J}$, Calandra T, et al. Bloodstream and endovascular infections due to Abiotrophia defectiva and Granulicatella species. BMC Infect Dis. 2006;6:9.

5. Govender S. Spinal infections. J Bone Joint Surg Br. 2005;87(11):1454-1458.

6. Rosenthal O, Woywodt A, Kirschner P, Haller H. Vertebral osteomyelitis and endocarditis of a pacemaker lead due to Granulicatella (Abiotrophia) adiacens. Infection. 2002;30(5):317-319.

7. Cassir N, Grillo JC, Argenson JN, Drancourt M, Levy PY. Abiotrophia defectiva knee prosthesis infection: A case report. J Med Case Rep. 2011;5:438.

8. Heath CH, Bowen SF, McCarthy JS, Dwyer B. Vertebral osteomyelitis and discitis associated with Abiotrophia adiacens (nutritionally variant streptococcus) infection. Aust N Z J Med. 1998;28(5):663.

9. Wilhelm N, Sire S, Le Coustumier A, Loubinoux J, Beljerd M, Bouvet A. First case of multiple discitis and sacroiliitis due to Abiotrophia defectiva. Eur J Clin Microbiol Infect Dis. 2005;24(1):76-78.

10. Uehara K, Chikuda H, Higurashi Y, Ohkusu K, Takeshita K, Seichi A, Tanaka S. Pyogenic discitis due to Abiotrophia adiacens. Int J Surg Case Rep. 2013;4(12):1107-1109. 